Running Head: Attendees Perception of Previous Professional Development Opportunities / Preferences for Follow-up Activities.

\title{
Professional Development: Attendees Perceptions from Previous PD Opportunities/ Preferences for Follow-up Activities
}

Jeffrey A. Knox, PhD and David L. Krupke, M.A.

St. Ambrose University

Davenport, lowa

Key Words: Professional Development, follow-up, attendees' perceptions, attendees' preferences 